\title{
Molecular mimicry between SARS-CoV-2 spike glycoprotein and mammalian proteomes: implications for the vaccine
}

\author{
Darja Kanduc $^{1}$ (1) $\cdot$ Yehuda Shoenfeld ${ }^{2,3}$
}

Received: 28 May 2020 / Accepted: 7 September 2020 / Published online: 18 September 2020

(C) Springer Science+Business Media, LLC, part of Springer Nature 2020

\section{Introduction}

The ethiopathology of the diseasome induced by the SARSCoV-2 infection in the human host [1] is under intensive investigation. A likely mechanism is that the multitude of the diseases encompassed within COVID-19 derives from molecular mimicry phenomena between the virus and human proteins [2]. The rationale is that, following an infection, the immune responses raised against the pathogen can crossreact with human proteins that share peptide sequences (or structures) with the pathogen, in this way, leading to harmful autoimmune pathologies $[3,4]$. Accordingly, lungs and airways dysfunctions associated with SARS-CoV-2 infection might be explained by the sharing of peptides between SARS-CoV-2 spike glycoprotein and alveolar lung surfactant proteins [2]. In support of this thesis, additional reports [5-8] highlight molecular mimicry and cross-reactivity as capable of explaining the SARS-CoV diseases. Of special interest, crossreactive $\mathrm{T}$ cell recognition between circulating "common cold" coronaviruses and SARS-CoV-2 has been also suggested [9].

Electronic supplementary material The online version of this article (https://doi.org/10.1007/s12026-020-09152-6) contains supplementary material, which is available to authorized users.

Darja Kanduc

dkanduc@gmail.com

Yehuda Shoenfeld

Yehuda.Shoenfeld@sheba.health.gov.il

1 Department of Biosciences, Biotechnologies, and Biopharmaceutics, University of Bari, Via Orabona 4, 70125 Bari, Italy

2 Zabludowicz Center for Autoimmune Diseases, Sheba Medical Center, Tel-Aviv University School of Medicine, Tel-Hashomer, Israel

3 I.M. Sechenov First Moscow State Medical University of the Ministry of Health of the Russian Federation, Sechenov University, Moscow, Russia
In this scientific framework, this study comparatively analyzed the peptide sharing between SARS-CoV-2 and mammalian species. Our reasoning is that if it were true that molecular mimicry between SARS-CoV-2 and human proteins contributes to or causes COVID-19, then different levels/ patterns of molecular mimicry vs. the virus should characterize the various animal species. Indeed, scarce data exist to indicate that domestic animals, for instances dogs and cats, can either transmit the virus or develop the virus-associated diseasome [10]. In general, currently, the consensus remains that there is no evidence that infected pets are a source of SARS-CoV-2 infection for people or other pets $[11,12]$.

Based on this rationale and using hexa- and heptapeptides as sequence probes [13-15], the peptide overlap between SARS-CoV-2 spike glycoprotein and mammalian proteomes was analyzed.

\section{Methods}

Peptide sharing analyses have been extensively described elsewhere $[16,17]$. Briefly, SARS-CoV-2 spike glycoprotein (NCBI protein $\mathrm{Id}=\mathrm{QHD} 43416.1$ ) primary sequence was dissected into hexa- and heptapeptides offset by one residue (i.e., MFVFLV, FVFLVL, VFLVLL, FLVLLP). We obtained 1268 hexapeptides and 1267 heptapeptides. Then each viral hexa- or heptapeptide was analyzed as a probe to scan for occurrences of the same hexa- or heptapeptide in the reference proteome from the following mammalian organisms (with taxonomy ID in parentheses): human, Homo sapiens (9606); mouse, Mus musculus (10090); rat, Rattus norvegicus (10116); cat, Felis catus (9685); dog, Canis lupus familiaris (9615); rabbit, Oryctolagus cuniculus (9986); chimpanzee, Pan troglodytes (9598); gorilla, Gorilla gorilla gorilla (9595); and rhesus macaque, Macaca mulatta (9544). Three viral proteomes were added as coronavirus controls: human coronavirus HKU1 (290028); human coronavirus 229E (11137); and human coronavirus OC43 (31631). The hexa/ 
Fig. 1 Peptide sharing between SARS-CoV-2 spike glycoprotein and mammalian and coronavirus proteomes. a Peptide sharing at the 6-mer level. b Peptide sharing at the 7-mer level

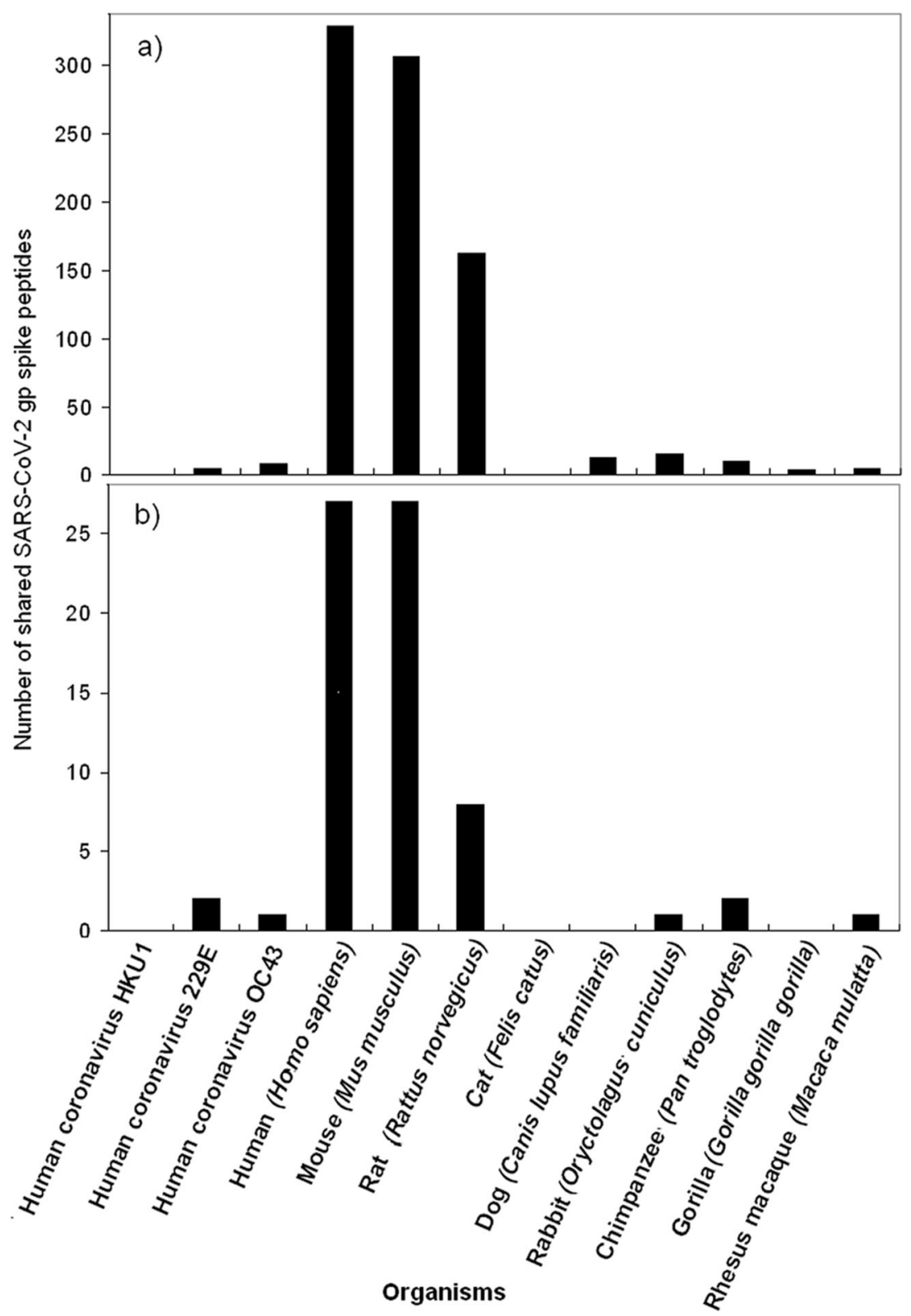

heptapeptide matching analyses were conducted by using Pir Peptide Matching program [18].

The expected value for hexapeptide sharing between two proteins was calculated by considering the number of all possible hexapeptides. Since in a hexapeptide, each residue can be any of the 20 amino acid (aa), the number of all possible hexapeptides $N$ is given by $N=20^{6}=64 \times 10^{6}$. Then, the number of the expected occurrences is directly proportional to the number of hexapeptides in the two proteins and inversely proportional to $N$. Assuming that the number of hexapeptides in the two proteins is $<<N$ and neglecting the relative abundance of aa, we obtain a formula derived by approximation, where the expected number of hexapeptides is $1 / N$ or $20^{-6}$. By applying the same calculation, the expected value for heptapeptide sharing between two proteins is equal to $20^{-7}$.

\section{Results}

The graphical illustration of the peptide sharing between SARS-CoV-2 spike glycoprotein and the analyzed mammalian and coronavirus proteomes is reported in Fig. 1. The hexaand heptapeptide sequences involved in the sharing are detailed in Tables S1 and S2, respectively.

Figure 1 shows that:

- A massive heptapeptide sharing exists between SARSCoV-2 spike glycoprotein and human proteins. Such a peptide commonality is unexpected and highly improbable from a mathematical point of view, given that, as detailed under the "Methods" section, the probability of the occurrence in two proteins of just one heptapeptide is 
equal to $\sim 20^{-7}$ (or 1 out of $1,280,000,000$ ). Likewise, the probability of the occurrence in two proteins of just one hexapeptide is close to zero by being equal to $\sim 20^{-6}$ (or 1 out of $64,000,000$ ).

- Only the viral peptide sharing with the murine proteome and, at a lesser extent, with the rat proteome keeps up with that shown by human proteins;

- Domestic animals, rabbit, and the three primates analyzed here have no or only a few peptide commonalities;

- Likewise, the proteomes of the three human coronaviruses HKU1, 229E, and OC43, which were used as viral controls, have no or only a few peptides in common with the spike glycoprotein. In this regard, it seems that the SARSCoV-2 spike glycoprotein is phenetically more similar to humans and mice than to its coronavirus "cousins".

\section{Conclusions}

This study thoroughly quantifies the hexa- and heptapeptide sharing of SARS-CoV-2 spike glycoprotein - which is a major antigen of the virus - with mammalian proteomes. A massive peptide commonality is present with humans and mice, i.e., organisms that undergo pathologic consequences following SARS-CoV-2 infection. Instead, no or a lowest number of common peptides are present in mammals that have no major pathologic sequelae once infected by SARS-CoV-2 [10-12]. Hence, the data appear to be an indisputable proof in favor of molecular mimicry as a potential mechanism that can contribute to or cause the SARS-CoV-2 associated diseases [8].

As a second relevant annotation, this study indicates that particular attention has to be dedicated to the choice of the laboratory animals to be used in preclinical studies during the formulation/validation of anti-pathogen vaccines. In the case in object, given the lowest level of sequence similarity of SARS-CoV-2 spike glycoprotein vs. primates proteins, results obtained in studies that use primates as animal models, i.e., rhesus macaque [19], would be unreliable because of the impossibility of verifying the occurrence of cross-reactivity and related autoimmunity in the absence of shared sequences. In this regards, data illustrated in Fig. 1 explain why, as highlighted by Hogan [20], "SARS-CoV infection of cynomolgus macaques did not reproduce the severe illness seen in the majority of adult human cases of SARS" [21]. Actually, no clinical signs of disease or marked lung pathology were seen in a study in which both rhesus and cynomolgus macaques were infected with SARS-CoV [22], and the Authors' conclusion is that the macaque model is of limited utility in the study of SARS and the evaluation of therapies. Likewise, McAuliffe et al. [23] described similar findings: "SARS-CoV administered intranasally and intratracheally to rhesus, cynomolgus and African Green monkeys replicated in the respiratory tract but did not induce illness".

As for domestic animals and cattles, coronaviruses are long known to be enteric pathogens of cats $(\mathrm{FeCoV}), \operatorname{dogs}$ (CaCoV), cattle (BCoV), and swine (TGEV) [24]. Nonetheless, coronaviruses do not appear to be pathogenic for domestic animals and cattles. Indeed, the scarce or null susceptibility to SARS-CoV-2-induced pathologies is certified by the American Veterinary Medical Association that verbatim declares: "during the first five months of the COVID-19 outbreak (January 1 - June 8, 2020), which includes the first twelve weeks following the March 11 declaration by the $\mathrm{WHO}$ of a global pandemic, fewer than 20 pets have tested positive, with confirmation, for SARS-CoV-2 globally. This despite the fact that as of June 8 , the number of people confirmed with COVID-19 exceeded 7 million globally and 1.9 million in the United States" (https://www. avma.org/).

In conclusion, in light of the data exposed in Fig. 1 and given the susceptibility parameters such as aging and health status, only aged mice appear to be a correct animal model for testing an anti-SARS-CoV-2 spike glycoprotein vaccine to be used in humans $[25,26]$.

Finally, this study once more reiterates the concept that only vaccines based on minimal immune determinants unique to pathogens and absent in the human proteome might offer the possibility of safe and efficacious vaccines [16, 27-30].

\section{Compliance with ethical standards}

Conflict of interest DK declares no conflicts. YS appears as a medical consultant in vaccine compensation court, USA.

\section{References}

1. Wang Z, Yang B, Li Q, Wen L, Zhang R. Clinical features of 69 cases with Coronavirus Disease 2019 in Wuhan, China. Clin Infect Dis. 2020:ciaa272. https://doi.org/10.1093/cid/ciaa272.

2. Kanduc D, Shoenfeld Y. On the molecular determinants of the SARS-CoV-2 attack. Clin Immunol. 2020;215:108426. https:// doi.org/10.1016/j.clim.2020.108426.

3. Oldstone MB. Molecular mimicry: its evolution from concept to mechanism as a cause of autoimmune diseases. Monoclon Antib Immunodiagn Immunother. 2014;33:158-65. https://doi.org/10. 1089/mab.2013.0090.

4. Kanduc D. Peptide cross-reactivity: the original sin of vaccines. Front Biosci. 2012;4:1393-401. https://doi.org/10.2741/s341.

5. Vlasova AN, Zhang X, Hasoksuz M, Nagesha HS, Haynes LM, Fang Y, et al. Two-way antigenic cross-reactivity between Severe Acute Respiratory Syndrome Coronavirus (SARS-CoV) and group 1 animal CoVs is mediated through an antigenic site in the Nterminal region of the SARS-CoV nucleoprotein. J Virol. 2007;81:13365-77. https://doi.org/10.1128/JVI.01169-07.

6. Cappello F. COVID-19 and molecular mimicry: the Columbus' egg? J Clin Neurosci. https://doi.org/10.1016/j.jocn.2020.05.015. 
7. Vojdani A, Kharrazianb D. Potential antigenic cross-reactivity between SARS-CoV-2 and human tissue with a possible link to an increase in autoimmune diseases. Clin Immunol. 2020;217:108480. https://doi.org/10.1016/j.clim.2020.108480.

8. Kanduc D. From anti-SARS-CoV-2 immune responses to COVID19 via molecular mimicry. Antibodies (Basel). 2020;9(3):E33. https://doi.org/10.3390/antib9030033.

9. Grifoni A, Weiskopf D, Ramirez SI, Mateus J, Dan JM, Rydyznski $\mathrm{C}$, et al. Targets of $\mathrm{T}$ cell responses to SARS-CoV-2 coronavirus in humans with COVID-19 disease and unexposed individuals. Cell. 2020. https://doi.org/10.1016/j.cell.2020.05.015.

10. Parry NMA. COVID-19 and pets: when pandemic meets panic. Forensic Sci Int. 2020;2:100090. https://doi.org/10.1016/j.fsir. 2020.100090.

11. Almendros A. Can companion animals become infected with Covid-19? Vet Rec. 2020;186:388-9. https://doi.org/10.1136/vr. m1194.

12. Li X. Can cats become infected with Covid-19? Vet Rec. 2020;186: 457-8. https://doi.org/10.1136/vr.m1455.

13. Pieczenik G. Are the universes of antibodies and antigens symmetrical? Reprod BioMed Online. 2003;6:154-6. https://doi.org/10. 1016/s1472-6483(10)61702-6.

14. Kanduc D. Homology, similarity, and identity in peptide epitope immunodefinition. J Pept Sci. 2012;18:487-94. https://doi.org/10. 1002/psc.2419.

15. Kanduc D. Pentapeptides as minimal functional units in cell biology and immunology. Curr Protein Pept Sci. 2013;14:111-20. https://doi.org/10.2174/1389203711314020003.

16. Kanduc D. Immunogenicity, immunopathogenicity, and immunotolerance in one graph. Anti Cancer Agents Med Chem. $2015 ; 15: 1264-8$. https://doi.org/10.2174/ 1871520615666150716105543 .

17. Kanduc D, Shoenfeld Y. Inter-pathogen peptide sharing and the original antigenic sin: solving a paradox. Open Immunol J. 2018;8:16-27. https://doi.org/10.2174/1874226201808010016.

18. Chen C, Li Z, Huang H, Suzek BE, Wu CH, UniProt Consortium. A fast peptide match service for UniProt knowledgebase. Bioinformatics. 2013;29:2808-9. https://doi.org/10.1093/ bioinformatics/btt484.

19. Yu J, Tostanoski LH, Peter L, Mercado NB, McMahan K, Mahrokhian SH, et al. DNA vaccine protection against SARSCoV-2 in rhesus macaque. Science. 2020:eabc6284. https://doi. org/10.1126/science.abc6284.

20. Hogan RJ. Are nonhuman primates good models for SARS? PLoS Med. 2006;3:e411. https://doi.org/10.1371/journal.pmed.0030411.
21. Lawler JV, Endy TP, Hensley LE, Garrison A, Fritz EA, Lesar M, et al. Cynomolgus macaque as an animal model for severe acute respiratory syndrome. PLoS Med. 2006;3:e149. https://doi.org/10. 1371/journal.pmed.0030149.

22. Rowe T, Gao G, Hogan RJ, Crystal RG, Voss TG, Grant RL, et al. Macaque model for severe acute respiratory syndrome. J Virol. 2004;78:11401-4. https://doi.org/10.1128/JVI.78.20.1140111404.2004.

23. McAuliffe J, Vogel L, Roberts A, Fahle G, Fischer S, Shieh WJ, et al. Replication of SARS coronavirus administered into the respiratory tract of African green, rhesus and cynomolgus monkeys. Virology. 2004;330:8-15. https://doi.org/10.1016/j.virol.2004.09. 030 .

24. Lowenstine LJ, Osborn KG. Respiratory system diseases of nonhuman primates. In: Abee CR, Keeling ME, Mansfield K, Tardif S, Morris T, editors. "Nonhuman Primates in Biomedical Research", Vol. 2: Diseases. Oxford: Academic Press, Elsevier; 2012. ISBN: 978-0-12-381366-4.

25. Roberts A, Paddock C, Vogel L, Butler E, Zaki S, Subbarao K. Aged BALB/c mice as a model for increased severity of severe acute respiratory syndrome in elderly humans. J Virol. 2005;79: 5833-8. https://doi.org/10.1128/JVI.79.9.5833-5838.2005.

26. Nagata N, Iwata-Yoshikawa N, Taguchi F. Studies of severe acute respiratory syndrome coronavirus pathology in human cases and animal models. Vet Pathol. 2010;47(5):881-92. https://doi.org/10. 1177/0300985810378760.

27. Kanduc D. Epitopic peptides with low similarity to the host proteome: towards biological therapies without side effects. Expert Opin Biol Ther. 2009;9:45-53. https://doi.org/10.1517/ 14712590802614041

28. Kanduc D. "Self-nonself" peptides in the design of vaccines. Curr Pharm Des. 2009;15:3283-9. https://doi.org/10.2174/ 138161209789105135.

29. Kanduc D, Shoenfeld Y. From HBV to HPV: designing vaccines for extensive and intensive vaccination campaigns worldwide. Autoimmun Rev. 2016;15:1054-61. https://doi.org/10.1016/j. autrev.2016.07.030.

30. Kanduc D. Hydrophobicity and the physico-chemical basis of immunotolerance. Pathobiology. 2020:1-9. https://doi.org/10. $1159 / 000508903$.

Publisher's note Springer Nature remains neutral with regard to jurisdictional claims in published maps and institutional affiliations. 